\title{
Lengua negra pilosa asociada a carcinoma escamocelular de esófago
}

\section{Black hairy tongue associated with squamous cell carcinoma of the esophagus}

\author{
Camilo de Jesús Blanco, ${ }^{*}$ (르 Andrés Fernando Blanco. ${ }^{2}$ (b)
}

\author{
Gacceso abierto \\ Citación: \\ Blanco CJ, Blanco AF. Lengua negra pilosa \\ asociada a carcinoma escamocelular \\ de esófago. Rev Colomb Gastroenterol. \\ 2021;36(Supl.1):112-115. https://doi. \\ org $/ 10.22516 / 25007440.623$ \\ Médico, Especialista en Cirugía Gastrointestinal \\ y Endoscopia Digestiva. Magíster en Educación. \\ Miembro Grupo Investigación, Grupo Educación \\ e Investigación, Universidad El Bosque, \\ Minciencias. Unidad de Videoendoscopia del \\ Restrepo Ltda., Bogotá, Colombia. \\ 2 Odontólogo, servicio social obligatorio. BITER \\ 13. Ejército Nacional de Colombia. Unidad de \\ Videoendoscopia del Restrepo Ltda., Bogotá, \\ Colombia. \\ *Correspondencia: Camilo Blanco. \\ camiloblancoa@gmail.com \\ Fecha recibido: $21 / 07 / 20$ \\ Fecha aceptado: 02/09/20

\section{(c) $(1)$}

\begin{abstract}
Resumen
La lengua negra pilosa (LNP) es una alteración benigna caracterizada por coloración oscura e hipertrofia con hiperqueratosis en las papilas filiformes de la superficie de la lengua. Han sido implicados varios factores intrínsecos y extrínsecos como causales, entre ellos la pobre higiene dental, el tabaquismo, la ingesta de bebidas oscuras, el uso de antibióticos y algunas patologías malignas. Reportamos el caso de un paciente con carcinoma escamocelular avanzado de esófago medio conjunto con LNP, asociación no encontrada previamente en la literatura.
\end{abstract}

\section{Palabras clave}

Lengua hiperpigmentada, lengua negra pilosa, lengua negra vellosa, lengua negra, pigmentación lingual.

\section{Abstract}

Black hairy tongue $(\mathrm{BHT})$ is a benign disorder characterized by dark coloration and hypertrophy with hyperkeratosis in filiform papillae on the surface of the tongue. Several intrinsic and extrinsic factors, including poor dental hygiene, smoking, drinking dark beverages, indiscriminate use of antibiotics, and some malignant diseases, have been proposed as potential causes. This is the case of a patient with advanced squamous cell carcinoma of the mid esophagus in conjunction with $\mathrm{BHT}$, a previously unknown association.

\section{Keywords}

Black hairy tongue; Black tongue; Hyperpigmentation of the tongue; Lingual pigmentation.

\section{INTRODUCCIÓN}

La lengua negra pilosa (LNP) es una condición benigna en la que el centro de la lengua cambia de coloración, desde negra hasta amarilla, con aspecto velludo ("peludo") por hipertrofia y elongación de las papilas filiformes (1). Su prevalencia es variable y está asociada a múltiples factores como pobre higiene oral; uso de tabaco; uso de antibióticos: penicilina, eritromicina, doxiciclina (2), linezolid (3), tetraciclina (4); y enjuagues bucales irritantes (5). Se ha descrito la asociación entre LNP y enfermedades sistémicas tales como VIH y malignidades (6). Se presenta el caso de una mujer adulta mayor con carcinoma escamocelular de esófago medio y, como hallazgo asociado, incidental, poco estético, pero no sintomático de LNP.

\section{PRESENTACIÓN DEL CASO CLÍNICO}

Paciente femenina, de 78 años, natural de Ubalá, Cundinamarca; procedente de Bogotá. Antecedente de 
hipotiroidismo tratado con levotiroxina y de hipertensión arterial en tratamiento con enalapril. Asiste a endoscopia digestiva por 1 mes de disfagia con sensación de atoramiento retroesternal y pérdida de $5 \mathrm{~kg}$ de peso. Menciona tener lengua negra desde hace 7 años, condición que no fue atendida en varias consultas.

$\mathrm{Al}$ examen físico se encuentra con aceptables condiciones generales; signos vitales: presión arterial (PA), 145/85 $\mathrm{mm} \mathrm{Hg}$; frecuencia cardíaca (FC), 76/min; frecuencia respiratoria (FR), 18/min; saturación de oxígeno $\left(\mathrm{SatO}_{2}\right)$, 92\%; fracción de oxígeno del aire inspirado $\left(\mathrm{FiO}_{2}\right), 24 \%$;

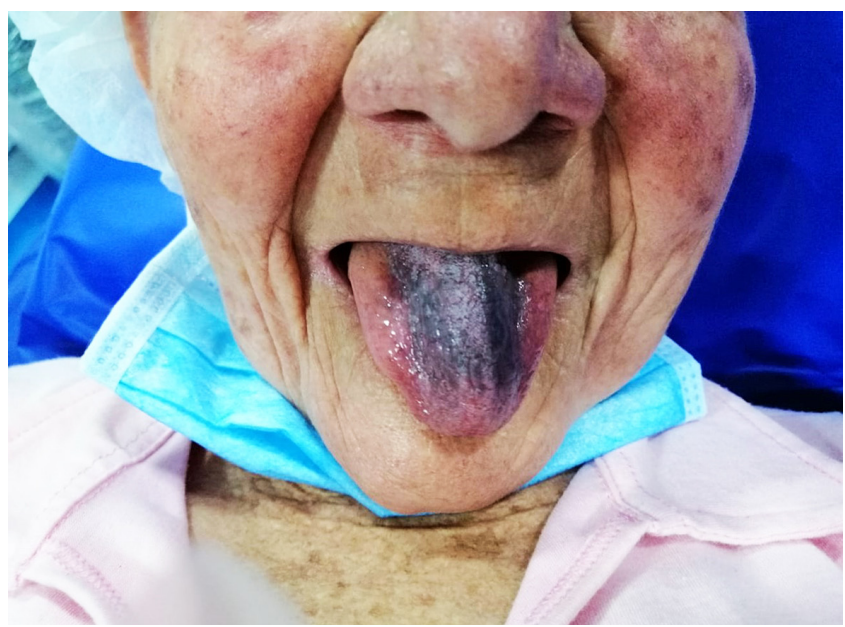

Figura 1. Paciente con coloración negra del dorso de la lengua, que no compromete los bordes laterales ni la punta.

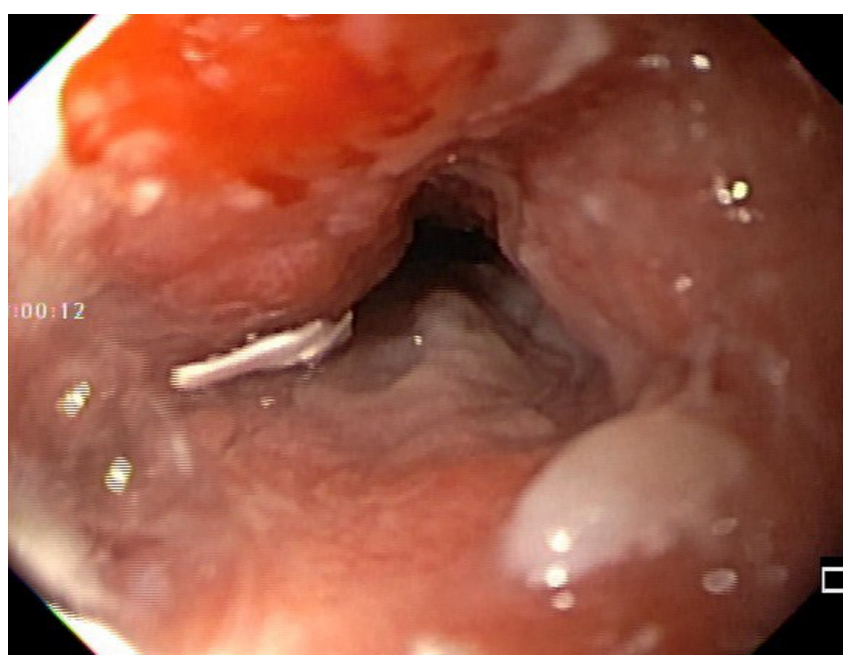

Figura 3. Endoscopia. Borde proximal de carcinoma escamocelular de esófago medio que compromete el $80 \%$ de la pared y de la luz. peso, $40 \mathrm{~kg}$; talla $1,5 \mathrm{~m}$; índice de masa corporal (IMC), $17,78 \mathrm{~kg} / \mathrm{m}^{2}$; y temperatura, $36^{\circ} \mathrm{C}$.

Durante la endoscopia se encuentra lengua de coloración oscura negruzca en su dorso, con aspecto de "pelos", sin compromiso en los bordes ni en la punta, con zona de despigmentación blanquecina en la zona central posterior (Figuras 1 y 2). En el esófago medio $(25 \mathrm{~cm})$, por paredes laterales derecha, izquierda y anterior, hay lesión nodular, infiltrante y friable que produce estenosis franqueable de $12 \mathrm{~mm}$ y llega hasta los $30 \mathrm{~cm}$, sin compromiso esofágico distal (Figuras 3 y 4 ).

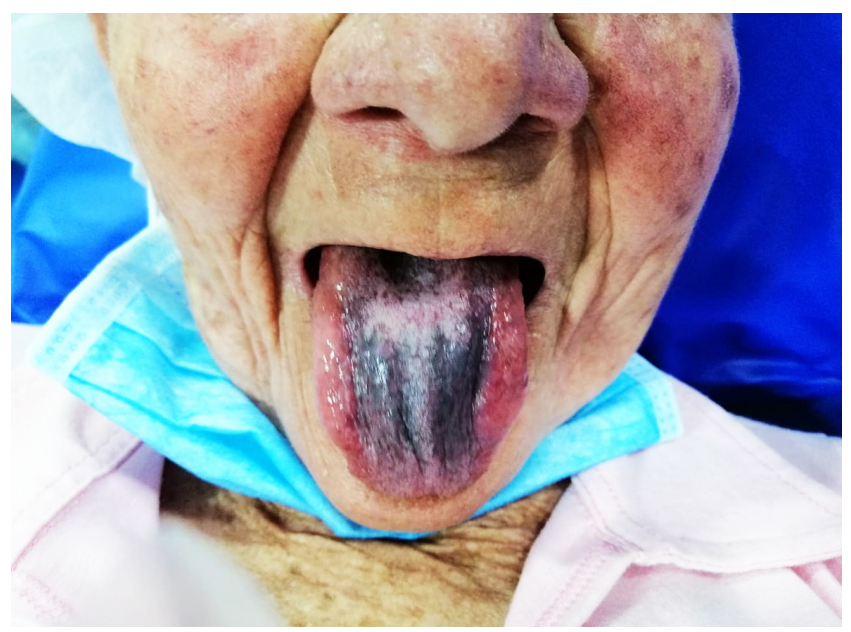

Figura 2. Paciente con lengua negra pilosa (LNP), con despigmentación blanquecina en la parte central posterior.

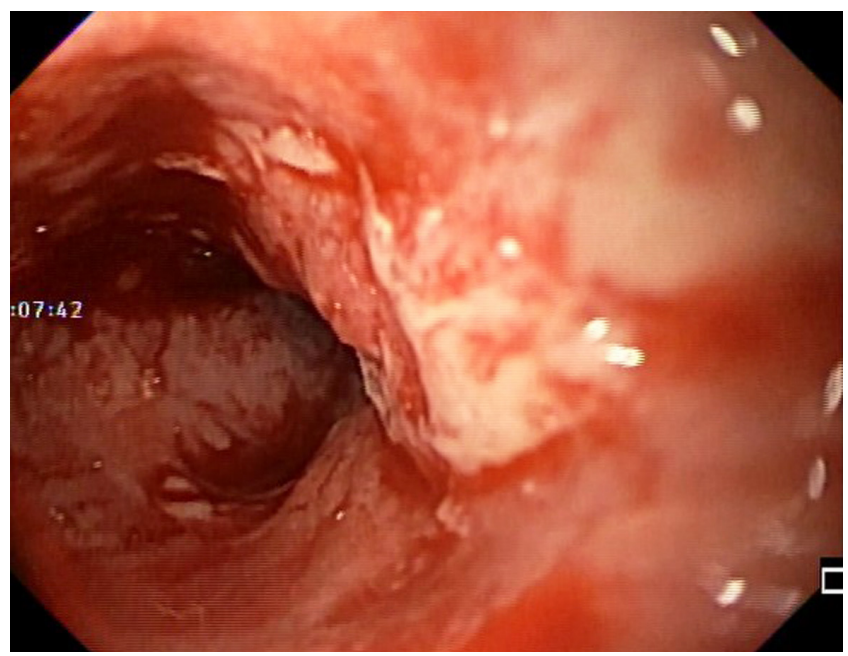

Figura 4. Endoscopia. Borde distal del carcinoma de esófago medio. 
Patología reportó carcinoma de células escamosas grandes, no queratinizante, infiltrante, pobremente diferenciado y ulcerado.

Al momento de esta publicación, la paciente está en estudios de extensión para determinar el tratamiento y en manejo orientado por odontología de su LNP.

\section{DISCUSIÓN}

La lengua negra pilosa (LNP) es una condición benigna que consiste en el oscurecimiento y apariencia velluda ("peluda") del dorso de la lengua, descrita por primera vez por Amatus Lusitanus en 1597 (7, 8). Su prevalencia es variable, aunque en estudios de salud oral es tan alta como del 11,3\%, más común en hombres de la tercera edad, fumadores severos, consumidores de té negro y café $(9,10)$, VIH positivos, edéntulos, con cáncer de próstata o linfoma de células B (6), y en los pacientes en tratamiento con antibióticos (2-4). Clínicamente se observa una placa o membrana negra distribuida en el dorso de la lengua, sin compromiso lateral ni de su punta (Figura 1) y, como en este caso, sin compromiso en la parte central y posterior (Figura 2). El cambio de color más común es negro, pero varía a café, verde o amarillo (11).

La LNP es usualmente asintomática, aunque se han descrito síntomas como náuseas, pérdida del gusto, halitosis, quemadura o cosquilleo en la lengua (12), aunque la molestia principal es su desagradable aspecto estético (13).

El aspecto piloso se debe a la inadecuada descamación de queratina (hiperqueratosis) sobre las papilas filiformes, que se elongan (hasta $18 \mathrm{~mm} \times 2 \mathrm{~mm}$ ), el cambio de color por levaduras o bacterias anaeróbicas (como Porphyromonas gingivalis) (14), cromogénicas, fermentadoras de aminoácidos y productoras de porfirina, y las modificaciones resultantes de factores ambientales (tabaco, alcohol, enjuagues bucales oxidantes, antibióticos, antipsicóticos, bloqueadores de la bomba de protones, quimioterapéuticos, radioterapia, antiVIH y medicamentos predisponentes de xerostomía) (7), que se pueden asociar a alteraciones del microbioma oral presentes en infección por VIH, enfermedad del injerto contra el huésped, esclerosis lateral amiotrófica, neuralgia del trigémino o malignidades (11).

El diagnóstico diferencial es con "pseudo-LNP" como acantosis nigricans, máculas melanóticas linguales congénitas, nevos melanocíticos congénitos, leucoplaquia premaligna, carcinoma escamocelular e infección hipertrófica por virus de herpes simple (15).

El diagnóstico clínico se precisa extendiendo el interrogatorio sobre los factores predisponentes descritos. El raspado suave de la lengua con bajalenguas o con cepillo de dientes puede sugerir LNP si el pigmento se disipa; no siempre fácil, ya que puede requerir múltiples intentos (16). Usualmente no se toma biopsia, excepto cuando la lesión se torna atípica, refractaria al tratamiento o sintomática, que genere sospecha de malignidad o de enfermedad sistémica (17); sin embargo, cuando se toma, reporta hiperqueratosis y acumulación de paraqueratosis en la punta de las papilas filiformes (18). La herramienta diagnóstica es la dermoscopia, que identifica los cambios en la forma y el color de las papilas filiformes; también es útil en la evaluación del éxito terapéutico $(18,19)$.

La primera línea de tratamiento se basa en la suspensión de los medicamentos asociados al cuadro, la práctica de una higiene oral excelente, la suspensión de los hábitos predisponentes y la generosa cepillada o raspado de la lengua que promueva la descamación de las papilas (17). Se describe buena efectividad con aplicación tópica de peróxido de hidrógeno al 3\%; la discontinuación del uso de enjuagues bucales oxidantes; la adecuación de dentaduras en edéntulos (que promueve la abrasión mecánica de las papilas descamadas al masticar alimentos sólidos) (20). En LNP resistentes, las papilas pueden ser removidas con quemadura o electrodesecación con láser de dióxido de carbono (21). Los tratamientos de segunda línea, anecdóticos y sin evidencia incluyen retinoides orales, antimicóticos, antibióticos, solución de urea tópica o triamcinolona, ácido salicílico y violeta de genciana.

En la revisión de la literatura no encontramos un reporte de LNP con cáncer de esófago, de manera que esta constituye una asociación que debe tenerse en cuenta.

\section{REFERENCIAS}

1. Hamad Y, Warren DK. Black hairy tongue. N Engl J Med. 2018;379(10):e16. https://doi.org/10.1056/NEJMicm1800351

2. Sakaguchi K, Watari T. Tetracycline induced black hairy tongue. J Gen Fam Med. 2020;21:80-81.

https://doi.org/10.1002/jgf2.300
3. Braggio C, Bocchialini G, Ventura L, Carbognani P, Rusca M, Ampollini L. Linezolid-induced black hairy tongue. Acta Biomed. 2018;89(3):408-10. https://doi.org/10.23750/abm.v89i3.7060

4. Poulopoulos AK, Antoniades DZ, Epivatianos A, Grivea IN, Syrogiannopoulos GA. Black hairy tongue in a 2-month-old 
infant. J Paediatr Child Health. 2008;44:377-9. https://doi.org/10.1111/j.1440-1754.2008.01307.x

5. Del Barrio P, Meza R, Vera C. Black Hairy Tongue. J Gen Intern Med. 2017;32(11):1266. https://doi.org/10.1007/s11606-017-4087-x

6. Thompson D, Kessler T. Drug-Induced Black Hairy Tongue. Pharmacotherapy. 2010;30(6):585-93. https://doi.org/10.1592/phco.30.6.585

7. Schlager E, Claire C, Ashack K, Khachemoune A. Black Hairy Tongue: Predisposing Factors, Diagnosis, and Treatment. Am J Clin Dermatol. 2017;18(4):563-9. https://doi.org/10.1007/s40257-017-0268-y

8. Jhaj R, Gour PR, Asati DP. Black hairy tongue with a fixed dose combination of olanzapine and fluoxetine. Indian J Pharmacol. 2016;48(3):318-20. https://doi.org/10.4103/0253-7613.182894

9. Avcu N, Kanli A. The prevalence of tongue lesions in 5150 Turkish dental outpatients. Oral Dis. 2003;9(4):188-95. https://doi.org/10.1034/j.1601-0825.2003.02933.x

10. Cariati P, Martínez L, Marín A, Julia M. Manejo clínico de la lengua negra vellosa. Emergencias. 2019;31(4):291-6.

11. Gurvits GE, Tan A. Black hairy tongue syndrome. World J Gastroenterol. 2014;20(31):10845-50. https://doi.org/10.3748/wjg.v20.i31.10845

12. Arab J, Vargas J, Morales C, Arrese M. Black hairy tongue during interferon therapy for hepatitis $\mathrm{C}$. Annals Hepatology. 2015;14(3):414-5. https://doi.org/10.1016/S1665-2681(19)31282-7

13. Nisa L, Giger R. Black hairy tongue. Am J Med. 2011;124(9):816-7. https://doi.org/10.1016/j.amjmed.2011.01.029
14. Fyrestam J, Bjurshammar N, Paulsson E, Mansouri N, Johannsen A, Ostman C. Influence of culture conditions on porphyrin production in Aggregatibacter actinomycetemcomitans and Porphyromonas gingivalis. Photodiagnosis Photodyn Ther. 2016;17:115-23. https://doi.org/10.1016/j.pdpdt.2016.11.001

15. Mangold AR, Torgerson RR, Rogers RS. Diseases of the tongue. Clin Dermatol. 2016;34(4):458-69. https://doi.org/10.1016/j.clindermatol.2016.02.018

16. Schwartz RH, Lee T. A two-week-old term baby with a black tongue. Clin Pediatr (Phila). 2015;54(11):1110-2. https://doi.org/10.1177/0009922814561354

17. Waldron CA, Shafer WG. Leukoplakia revisited. A clinicopathologic study 3256 oral leukoplakias. Cancer. 1975;36(4):1386-92. https://doi.org/10.1002/1097-0142(197510)36:4<1386::aidcncr2820360430>3.0.co;2-7.

18. Wang $\mathrm{X}$, Yang $\mathrm{S}$, Wang Y. Black hairy tongue in a patient receiving enteral feeding. Br J Dermatol. 2019; 181:ppe91. https://doi.org/10.1111/bjd.18205

19. Kobayashi K, Takei Y, Sawada M, Ishizaki S, Ito H, Tanaka $\mathrm{M}$. Dermoscopic features of a black hairy tongue in 2 Japanese patients. Dermatol Res Pract. 2010;145878. https://doi.org/10.1155/2010/145878

20. Villalobos A, Jiménez F. Lengua vellosa negra. Reporte de caso clínico. ODOVTOS-Int. J. Dent. Sc. 2016;18:29-33. http://dx.doi.org/10.15517/ijds.v0i0.24478

21. Jahanbani J, Sandvik L, Lyberg T, Ahlfors E. Evaluation of oral mucosal lesions in 598 referred Iranian patients. Open Dent J. 2009;3:42-7. https://doi.org/10.2174/1874210600903010042 